\title{
Identifying the research needs in the global assessment of toxic compounds 10 years after the signature of the Stockholm Convention
}

\author{
Gerhard Lammel • Rainer Lohmann
}

Received: 26 April 2012 / Accepted: 3 May 2012

(C) Springer-Verlag 2012

Persistent organic pollutants (POPs) are targeted by the Stockholm Convention for reduction and eventual elimination from production, trade and release. All POPs share properties that make them long lived (persistent), get enriched along the food chains (bioaccumulative), present at elevated concentrations in remote locations (prone to long-range transport) and elicit adverse effects (toxic). Since its adoption on 22 May 2001, the convention has identified just over 20 chemicals and groups of compounds as POPs. These include the original 'dirty dozen' compounds as the coming into force of the convention (aldrin, chlordane, dieldrin, endrin, heptachlor, hexachlorobenzene, mirex, toxaphene, DDT, polychlorinated dibenzo-p-dioxins and dibenzofurans (PCDD/Fs) and polychlorinated biphenyls), followed by another nine compounds in 2009 (chlordecone; hexabromobiphenyl; tetra-, penta-, hexa- and hepta bromodiphenylether;

Responsible editor: Philippe Garrigues

G. Lammel $(\bowtie)$

Research Centre for Toxic Compounds in the Environment,

Masaryk University,

Brno, Czech Republic

e-mail: lammel@recetox.muni.cz

G. Lammel

Max Planck Institute for Chemistry,

Mainz, Germany

R. Lohmann

Graduate School of Oceanography, University of Rhode Island,

Narragansett, RI, USA

e-mail: lohmann@gso.uri.edu $\alpha$-hexachlorocyclohexane; $\beta$-HCH; $\gamma$-HCH (lindane); pentachlorobenzene; perfluorooctane sulfonic acid, its salts and perfluorooctane sulfonyl fluoride). In 2011, endosulfan was added to the list.

Ten years later, after the adoption of the convention, an expert meeting was organized by the Regional Centre of the Stockholm Convention for capacity building and transfer of technology in Central and Eastern Europe, the Research Centre for Toxic Compounds in the Environment of Masaryk University, and held in Brno, Czech Republic, on May 22-24, 2011, with the support of the Secretariat of the Stockholm Convention (SSC), the Ministry of the Environment of the Czech Republic, the European Association for Chemical and Molecular Sciences-Division of Chemistry and the Environment and the American Chemical Society. The topics addressed were on aims and instruments of the convention and cooperation with related conventions (Rotterdam, Basel) and protocols, national policies and stakeholders, in particular science.

The workshop participants assessed progress with the Stockholm Convention and identified major knowledge gaps, challenges and research needs associated with the global assessment of hazardous chemicals. The expertise of 40 invited scientists from 16 countries of three UN regions was sought and supplemented by experience of the representatives of the SSC, UNEP, and government. Their expertise encompasses the fields of environmental chemistry and exposure modelling; monitoring of air, water and biota including human exposure, effects and risks; and data interpretation and management. The participants identified 10 major priority areas for future work (Klanova et al. 2011): 
1. Source control of POPs and other chemicals

2. Global scale transport, sources and sinks

3. Persistence of chemical compounds

4. Tools for monitoring of chemical concentrations and fluxes

5. Future trends and scenarios

6. Global monitoring plan

7. Global Earth Observation System of Systems (GEOSS)

8. Capacity building

9. Limiting adverse effects from hazardous chemicals

10. Effective science to serve policy needs

This special issue of ESPR compiles contributions from workshop participants that highlight the current scientific knowledge of POPs and the implementation of the SC, knowledge gaps and future research directions.

In more detail, the contributions of Dvorská et al., Aliyeva et al., Adu-Kumi et al., Cecinato et al. and Mosca et al. report on case studies addressing various classes of legacy POPs and/or emerging contaminants in polluted environments of Armenia, Azerbaijan, Ghana and Italy (two papers), respectively. The paper by Mikeš et al. reports on long-term monitoring of POPs in breast milk from the Czech Republic. The papers by Vicaretti et al. and Wong et al. focus on key processes of POP cycling, i.e. emissions (of PCDD/Fs from waste incineration) and air-sea exchange (of $\alpha-\mathrm{HCH}$ ), respectively. Stemmler and Lammel study longterm cycling of PCB on the regional scale with a multicompartment chemistry-transport model. The contributions of Balducci et al. are a review of analytical techniques for the quantification of a wide spectrum of POPs and emerging contaminants in atmospheric matrices, and the paper of Lohmann et al. is a review on the state of the art with regard to passive sampling techniques in aqueous matrices.

\section{Reference}

Klánová J, Diamond ML, Jones KC, Lammel G, Lohmann R, Pirrone $\mathrm{N}$, Scheringer $\mathrm{M}$ et al (2011) Identifying the research and infrastructure needs for the global assessment of hazardous chemicals 10 years after establishing the Stockholm Convention. Environ Sci Technol 45:7617-7619

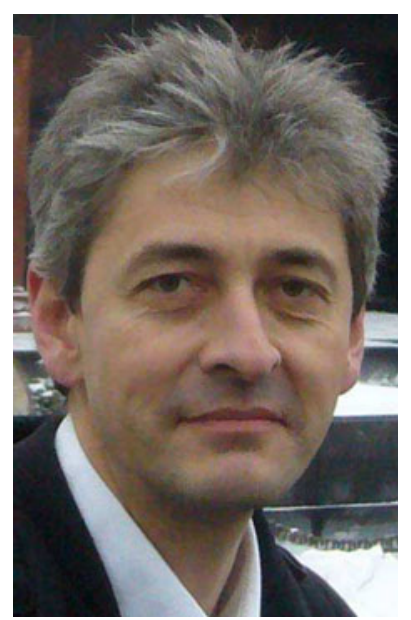

Gerhard Lammel is a full professor at the Masaryk University, Faculty of Science, Brno, Czech Republic and senior scientist at the Max Planck Institute for Chemistry, Mainz, Germany. He earned his $\mathrm{PhD}$ in Atmospheric Chemistry (1988) from the University of Mainz / Max Planck Institute for Chemistry and his teaching degree (Habilitation, 2000) for Environmental Chemistry from the University of Hohenheim, Stuttgart, Germany. In his research he has been focusing on aerosol chemistry through field and laboratory experimental studies and multicompartmental chemistry through modelling and field studies. He has published more than 70 papers on atmospheric and multicompartmental chemistry in international scientific journals and about 60 other contributions. He is a member of the Division on Chemistry and the Environment of EuCheMS (DCE, since 2004) and has been serving in various committees, the board of the Environmental Chemistry and Ecotoxicology branch of the German Chemical Society (GDCh, chair 2007-2010), besides other. He is member of the editorial boards of "Environmental Science and Pollution Research" and "Atmospheric Environment".

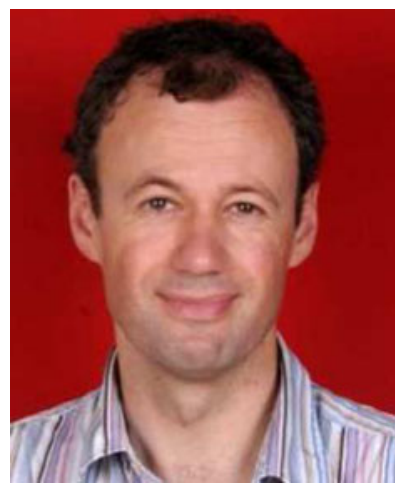

Dr Rainer Lohmann is currently an associate professor of oceanography at the University of Rhode Island's Graduate School of Oceanography. He obtained his $\mathrm{PhD}$ in Environmental Science (2000) at Lancaster University (UK). His areas of expertise include the global transport and fate of POPs with a focus on the role of oceans, and the use of passive samplers to detect organic pollutants and predict their bioaccumulation. At URI, he teaches "Marine Pollution" and "Marine \& Environmen-

tal Organic Chemistry". He has published over 60 papers on atmospheric, oceanic and environmental chemistry at leading international journals. He received the Roy F. Weston Environmental Chemistry Award by the Society of Environmental Toxicology and Chemistry (SETAC), and has been awarded fellowships from the Alexander-von-Humboldt Society in 2011, the Research Center for Ocean Margins (Bremen) in 2004, and the German Academic Exchange Service in 2000. He serves as editor for the journal "CLEAN - Air, Soil and Water'. 